\title{
Evaluation of the Performance of Nitrate Reductase Assay for Rapid Drug-susceptibility Testing of Mycobacterium tuberculosis in North India
}

\author{
Anamika Gupta, Malay Ranjan Sen, Tribhuban Mohan Mohapatra, and Shampa Anupurba \\ Department of Microbiology, Institute of Medical Sciences, Banaras Hindu University, Varanasi 22I 005, India
}

\begin{abstract}
The objective of the study was to evaluate the performance of nitrate reductase assay (NRA) as a rapid, reliable and inexpensive method for drug-susceptibility testing (DST) of Mycobacterium tuberculosis against firstline antitubercular drugs, such as rifampicin (RIF), isoniazid (INH), streptomycin (STR), and ethambutol (EMB). In total, 286 isolates were subjected to test by proportion method (PM) and NRA. By comparing the results of NRA with those of the gold standard PM, sensitivities and specificities were $98.4 \%, 97 \%, 88.5 \%$, and $94.2 \%$ and $100 \%, 100 \%, 94 \%$, and $99 \%$ for RIF, INH, STR, and EMB respectively. The positive predictive values were $100 \%, 100 \%, 95 \%$, and $98 \%$ for RIF, INH, STR, and EMB respectively. The negative values were $99 \%, 98 \%, 87 \%$, and $96 \%$ for RIF, INH, STR, and EMB respectively. The median time of obtaining results was shorter using NRA (10 days) compared to PM (28 days). An excellent agreement was observed between the two phenotypic tests with the $\kappa$ values of $0.98,0.97,0.81$, and 0.93 for RIF, INH, STR, and EMB respectively. The results demonstrated that NRA is suitable for the early determination of INH and RIF resistance and has the potential to be a useful tool for rapid drug-sensitivity test of M. tuberculosis in resource-constrained settings.
\end{abstract}

Key words: Antitubercular agents; Mycobacterium tuberculosis; Drug resistance, Mutiple; Nitrate reductase assay; Tuberculosis, Multi-drug-resistant; India

\section{INTRODUCTION}

Tuberculosis (TB) remains the greatest cause of illness and death worldwide, especially in Asia and Africa, which have accounted for $55 \%$ and $31 \%$ of the cases respectively in 2007 (1).

The first five countries in terms of total numbers of TB cases in 2007 were India ( 2 million), China (1.3 million), Indonesia (0.53 million), Nigeria (0.46 million), and South Africa (0.46 million). Of the 9.27 million incidences of TB cases throughout the world in 2007, an estimated 1.37 million (15\%) were HIV-positive; $79 \%$ of these HIV-positive cases were in the African region, and $11 \%$ were in the South-East Asia region. Developing countries ac-

Correspondence and reprint requests should be addressed to:

Prof. S. Anupurba

Department of Microbiology

Institute of Medical Sciences

Banaras Hindu University

Varanasi 221005

India

Email: shampa_anupurba@yahoo.co.in

Fax: +91 5422367568 count for $95 \%$ of active TB cases and deaths due to TB worldwide (2). Malnutrition and poor sanitary conditions contribute to the large-scale occurrence of the disease. The HIV pandemic has also played a role in enhancing the burden of the disease all over the world (3).

In developing countries, many national TB-control programmes have failed because of their low casedetection rates, and once a case is detected, cure may also be difficult because of poor case management and inadequate control of drug prescription (4). Furthermore, the most worrisome trend in recent years, which challenged the global prospects for TB control, is an increase in drug-resistant TB, particularly multidrug-resistant TB (MDR-TB), defined as resistance to at least rifampicin (RIF) and isoniazid (INH). Extensively drug-resistant TB (XDR-TB) occurs in MDR strains which are resistant to any fluoroquinolone and at least one of three injectable second-line aminoglycoside drugs, i.e. amikacin, kanamycin, or capreomycin. In response to the emergence of XDR-TB, the World Health Organization (WHO) is now calling for universal access to culture and DST by 2015 for all new 
pulmonary TB patients (5). According to the WHO, 500,000 new cases of MDR-TB occur globally every year, and MDR-TB has been reported in 2.9\% and $15.3 \%$ of new and previously-treated cases respectively (6).

There is an urgent demand for early and proper detection of MDR and XDR-TB cases for the effective management and control of TB. Conventional method, i.e. proportion method (PM), is the gold standard for DST of Mycobacterium tuberculosis (MTB) but has some limitations, such as cumbersomeness and long-turnaround time (TAT). In recent years, a multitude of techniques for rapid DST has been designed and evaluated, such as colorimetric redox method (7), radiometric method BACTEC 460-TB (8), commercial MGIT, E-tests $(9,10)$, and molecular methods-Genotype MTBDRplus and INNO-LiPA $(11,12)$. Most of these have proved their reliability and accuracy but are limited to developed countries only because of high expenses while others, such as colorimetric redox methods or nitrate reductase assay, need more evaluation in the field.

NRA —also called Griess method (13) — was first described by Angeby et al. (14). NRA is based on the principle that MTB has the capability of reducing nitrate to nitrite. The presence of nitrite can be detected by addition of Griess reagent which changes the colour of the culture medium (15). Several studies evaluated the performance of NRA but only a few were in India (16-21). The present study was, therefore, conducted to evaluate the performance of NRA compared to the gold standard PM for DST of MTB. Susceptibility tests were performed for all four first-line antitubercular drugs, i.e. RIF, INH, streptomycin (STR), and ethambutol (EMB).

\section{MATERIALS AND METHODS}

\section{Settings}

M. tuberculosis strains were isolated from clinical sputum specimens collected from three different TB centres of Varanasi and from specimens submitted to the Department of Microbiology, Institute of Medical Sciences, Banaras Hindu University, India, for 22 months during January 2008-October 2009.

\section{Mycobacterial strains}

In total, 286 strains of MTB were evaluated in the study. The isolates and sputum specimens were obtained from cases reported for pulmonary TB. H37Rv (ATCC 27294), and M. intracellulare (ATCC 13950) strains served as nitrate-positive and nitrate-negative controls respectively. A known MDR strain was also used as control. All the strains were first confirmed for their nitrate reductase activity (22), which is part of the routine biochemical tests. The strains were confirmed to be MTB based on their growth rates, pigmentation, colony morphology, and biochemical tests, such as heat-stable catalase, niacin accumulation, and susceptibility to $p$-nitrobenzoic acid (PNB) (22). All the strains were sub-cultured in Lowenstein-Jensen (LJ) medium for four weeks before NRA was performed.

\section{Antitubercular drugs}

INH, STR, RIF, and EMB were obtained in powder form from Sigma (St Louis, Missouri, USA). Each drug was prepared at a concentration of $10 \mathrm{mg} / \mathrm{mL}$ in sterile distilled water, except RIF, which was dissolved in dimethylformamide (DMF). Stock solutions were filter-sterilized and stored at $-20^{\circ} \mathrm{C}$ for not more than one month.

\section{Media}

Conventional LJ medium was prepared as described by Canetti et al. (23). The LJ medium for NRA was prepared with a slight modification: 1 $\mathrm{mg} / \mathrm{mL} \mathrm{KNO}_{3}$ was added to the $\mathrm{LJ}$ medium, with or without antibiotics, dissolved by stirring and then aliquoted and inspissated once for 50 minutes at $80^{\circ} \mathrm{C}$. For NRA, the bottles containing LJ with antibiotics and $\mathrm{KNO}_{3}$ were used in duplicates while control tubes were used in triplicate.

\section{Griess reagent}

Fifty percent (vol/vol) concentrated hydrochloric acid $(\mathrm{HCl}), 0.2 \%$ (wt/vol) sulphanilamide, and $0.1 \%$ (wt/vol) n-1-naphthylethylenediamine dihydrochloride were prepared in small volumes and were mixed shortly before use in the ratio: 1 part: 2 part: 2 part respectively.

\section{DST by PM}

The PM was carried out on the LJ medium according to the laboratory's standard procedures with the recommended critical concentrations of $40 \mu \mathrm{g} / \mathrm{mL}$ for RIF, $0.2 \mu \mathrm{g} / \mathrm{mL}$ for INH, $2 \mu \mathrm{g} / \mathrm{mL}$ for EMB, and 4 $\mu \mathrm{g} / \mathrm{mL}$ for STR $(23,24)$.

\section{DST by NRA}

NRA was performed according to the method described by Angeby et al. (14). Bacterial suspensions were made from a sub-cultured $\mathrm{LJ}$ tube by dispensing two $1-\mu \mathrm{L}$ loopfuls of bacteria in $0.5 \mathrm{~mL}$ of phosphate-buffered saline (PBS) (pH 7.4) in 7.5-mL screw-cap bottles containing a few 3-mm diameter glass beads and vortexed to obtain a uniform solution. To obtain the turbidity of McFarland standard no. 1, approximately $2.5 \mathrm{~mL}$ of PBS was added. Part 
of the suspension was diluted at 1:10 in PBS. For each strain, $0.2 \mathrm{~mL}$ of the undiluted suspension was inoculated into tubes containing the $\mathrm{LJ}$ medium with $\mathrm{KNO}_{3}$ and the antibiotics in duplicates to reduce the risk of contamination and to assess the reproducibility of the test while $0.2 \mathrm{~mL}$ of the $1: 10$ dilution was inoculated into three drug-free tubes containing $\mathrm{LJ}$ with $\mathrm{KNO}_{3}$. The latter tubes served as growth controls. The tubes were incubated at $37^{\circ} \mathrm{C}$. After seven days, $0.5 \mathrm{~mL}$ of Griess reagent our change could be observed, the corresponding antibiotic-containing tubes were also tested, and susceptibility results were read. If no colour change was observed in the growth control tube, this tube was discarded, and the other two control tubes and the antibiotic-containing tubes were re-incubated. The procedure was then repeated at day 10, using the second growth control, and if needed, also at day 14 , using the last growth control tube.

The results were classified as negative if no colour change of the medium was observed and positive if pink to violet colour appeared in the medium. An isolate was considered to be resistant to a certain drug if there was a colour change in the antibioticcontaining tube in question greater than that in the 1:10 diluted growth control on the same day.

\section{Analysis of data}

The MedCalc software (MedCalc, Belgium) was used for calculating the statistical parameters, such as sensitivity, specificity, and accuracy. The predictive values were calculated using the prevalence of RIF, INH, STR, and EMB resistance in all the TB cases in Varanasi, India. The agreement between the NRA and the standard PM was determined by the $\kappa$-statistic. The $\kappa$ value, a measure of test reliability, was interpreted as follows: $<0.2$ poor; $0.21-0.4$ fair; 0.41-0.6 moderate; 0.61-0.8 good; and $\geq 0.81$ excellent (25).

\section{RESULTS}

DST for the first-line antitubercular drugs, i.e. RIF, INH, STR, and EMB, was performed with 286 clinical MTB isolates. The results of 107,138 , and 41 isolates were obtained after 7,10 , and 14 days respectively and were compared with those produced by the gold standard PM. The results are shown in the Table. For RIF, 123 and 161 strains were detected to be resistant and susceptible respectively by means of both the methods while two false-negative results were produced with the NRA. For INH, both the methods detected 126 resistant strains and 156 susceptible strains; four false-negative results were obtained with the NRA. For EMB, 115 and 162 was added to one drug-free control tube. If any col-

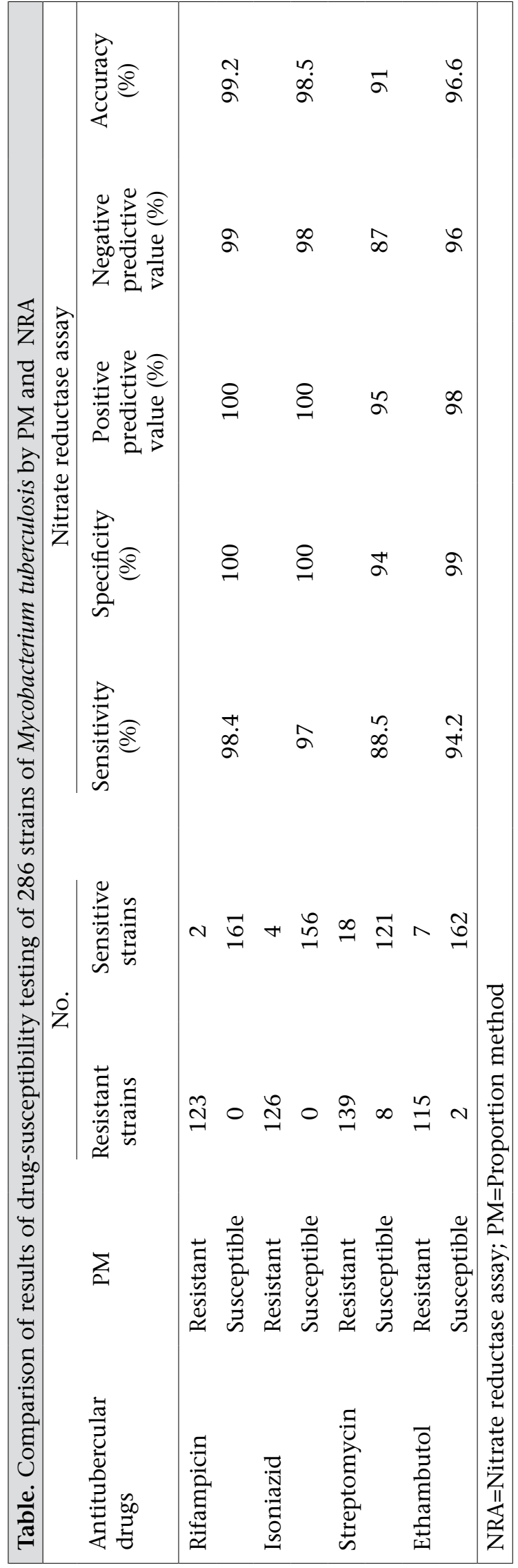

22 
strains were identified as true-positive and truenegative respectively by both the methods while 7 and 2 strains were misidentified as susceptible and resistant respectively by NRA. For STR, 139 isolates were correctly detected as resistant and 121 as sensitive by both the methods; 26 isolates gave discrepant results, 18 of which were false-negative i.e. resistant by PM while being susceptible by NRA, and 8 isolates were false-positive i.e. susceptible by PM but resistant by NRA. An excellent agreement was found between the two tests with the $\kappa$ values of $0.98,0.97,0.81$, and 0.93 for RIF, INH, STR, and EMB respectively. The sensitivity of the NRA compared to that of PM was observed to be $98.4 \%$, 97\%, 88.5\%, and 94.2\% for RIF, INH, STR, and EMB respectively. Specificities were 100\%, 100\%, 94\%, and 99\% for RIF, INH, STR, and EMB respectively. The positive predictive values were 100\%, 100\%, 95\%, and 98\% for RIF, INH, STR, and EMB respectively. The negative values were 99\%, 98\%, 87\%, and $96 \%$ for RIF, INH, STR, and EMB respectively. The accuracy of NRA was high with the PM for RIF (99.2\%), INH (98.5\%), and EMB (96.6\%), except for STR (91\%). An excellent agreement was observed in the results for RIF and INH. A good correlation was also found for EMB whereas some problems existed in detecting STR resistance and susceptibility (Table).

\section{DISCUSSION}

The most worrisome trend during recent years is an increase in multidrug-resistant (i.e. resistant to RIF and INH) TB strains. Rapid detection of MDR strains is very important to restrict their spread in the population. Current methods for DST of MTB are either costly or very slow. So, a cost-effective and rapid drug-susceptibility method is required to guide the treatment of TB. Results of meta-analysis of Martin et al. about NRA suggest that the NRA is highly sensitive and specific for determining RIFand INH-resistant TB in both culture isolates and directly on clinical sputum specimens (26). Most studies had a sensitivity of $95 \%$ or greater, and nearly all were $100 \%$ specific with high degree of accuracy. The average TAT was 5-12 days with indirect NRA and 14-21 days with direct NRA. The biggest asset of NRA is that there is no need to change the laboratory infrastructure as it is performed in the classical LJ medium, routinely used in TB laboratories, with the addition of $\mathrm{KNO}_{3}$. There is no need of any sophisticated equipment or expensive reagent, making it a widely-used method. Results are easy to observe by a colour change of the medium.

In the present study, an excellent agreement between the results of NRA and PM was observed with the $\kappa$ values of $0.98,0.97,0.81$, and 0.93 for RIF, INH, STR, and EMB respectively. RIF together with INH are the most important drugs for the treatment of TB. Sensitivities of NRA for RIF and INH were $98.4 \%$ and $97 \%$ respectively whereas specificity was $100 \%$ for both the drugs. However, an acceptable result of NRA was also found for EMB, with sensitivity and specificity of $94.2 \%$ and $99 \%$ respectively but some problems existed in detecting STR resistance. Other studies have also reported high sensitivity and specificity values for INH and RIF $(14,27)$.

The accuracy with the PM was high for all the drugs used in this study, except STR which does not come under the standard level, according to the criteria established by the WHO/IUATLD Supranational Laboratory Network, which has proposed the accuracy levels of $99.0 \%$ and $97.0 \%$ for RIF and INH respectively and $92.0 \%$ for EMB and STR as reasonable performance goals for reference laboratories. Although it has been already reported that STR is a difficult drug to test even by standard methods (28), the performance of NRA for STR was not acceptable because the expected level of agreement (92\%) was not achieved. However, the $\kappa$ value of the tests for STR showed that there is an excellent agreement $(0.81)$ between the two phenotypic tests.

The performance of NRA was evaluated in a multicentre study by Martin et al. to ascertain the susceptibility of MTB against first-line antitubercular drugs (29). The accuracy was greater than $97 \%$ for INH, EMB, and RIF while that for STR was inferior (85.3\%). In another study, Martin et al. reported the evaluation of NRA for ofloxacin, a second-line drug, and found complete agreement with the agar PM (30). Therefore, NRA has the capability to be used also for the evaluation of second-line drugs. In addition, Lemus et al. evaluated indirect NRA with 320 strains of MTB and found an overall agreement of $98.8 \%$ between the NRA and the PM (31).

Recently, Khan and Sarkar have developed a dormant stage-specific anti-TB screening protocol in microplate format using Wayne's hypoxic model and nitrate reductase activity in M. bovis BCG (Bacillus Calmette-Guérin) culture (32). They concluded that the assay provides an easy, inexpensive, rapid, robust and high-content screening tool to search novel anti-TB molecules against both active and dormant bacilli with signal to noise ratio and $\mathrm{Z}$ factor, i.e. 8.5 and 0.81 respectively.

With the objective of reducing the TAT of DST by omitting the pre-isolation step, the NRA has been applied directly on clinical sputum specimens. A full agreement was observed for the detection of 
RIF resistance while some discordant results were obtained for other drugs (33). Visalakshi et al. observed sensitivity and specificity of the direct NRA and indirect PM to be $94 \%$ and $98 \%$, and $100 \%$ and 98\% for RIF and INH respectively with an excellent agreement between the two tests (21). Moreover, Shikama et al. stated 100\% sensitivity and specificity of NRA for RIF (34). In another study of Shikama et al., reproducibility of NRA was $100 \%$ for INH and EMB and 97\% for STR and RIF, with 98.3\% agreement between the results of NRA and PM for INH and RIF (35).

Syre et al. used colorimetric nitrate reductase-based antibiotic-susceptibility (CONRAS) test for DST of MTB against INH and RIF in liquid cultures (36). The results were produced within five days, indicating that the CONRAS test is an alternative in all settings, particularly for resource-poor countries.

Being advantageous, NRA has some limitations, such as some strains $(<1 \%)$ of MTB lack nitrate reductase (37) rendering the test invalid. However, in our setting, we could not find any such strain in routine biochemical tests, including nitrate reductase test. In addition, nitrate might be reduced to nitric oxide beyond nitrite which cannot be detected by Griess reagent. Hence, zinc-dust was added to all negative tubes (22). Zinc reduces nitrate rapidly, and a true-negative test will directly turn red while there will be no change in colour in a tube where reduction has passed beyond nitrite. Another possible limitation of NRA is that it can give positive results with atypical mycobacteria $M$. kansasii, $M$. szulgai, M. flavescens, M. terrae complex, and some rapid growers (37) while $M$. bovis is nitrate-negative.

\section{Conclusions}

Our results indicate that NRA is suitable for the early determination of INH and RIF resistance. On the basis of the findings, we conclude that NRA has the potential to be a useful tool for rapid DST of MTB in resource-poor countries with limited laboratory facilities because of its low-cost, rapidness, reproducibility of results, simplicity and lack of requirement of expensive reagents and equipment.

\section{ACKNOWLEDGEMENTS}

The authors thank Dr. Juan Carlos Palomino for providing the Medcalc software through e-mail. The authors are also thankful to Dr. VL Nag and Dr. G Nataraj for reference strains.

\section{REFERENCES}

1. World Health Organization. Global tuberculosis control: surveillance, planning, financing. Geneva:
World Health Organization, 2008:19. (http://www. who.int/tb/publications/global_report/2008/pdf/ fullreport.pdf, accessed on 28 December 2009).

2. World Health Organization. Global tuberculosis control: epidemiology, strategy, financing. WHO report 2009. Geneva: World Health Organization, 2009:1. (WHO/HTM/TB/2009.411).

3. Corbett EL, Marston B, Churchyard GJ, De Cock KM. Tuberculosis in sub-Saharan Africa: opportunities, challenges, and change in the era of antiretroviral treatment. Lancet 2006;367:926-37.

4. Guwatudde D, Zalwango S, Kamya MR, Debanne SM, Diaz MI, Okwera A et al. Burden of tuberculosis in Kampala, Uganda. Bull World Health Organ 2003;81:799-805.

5. World Health Organization. The global plan to stop TB 2006-2015: actions for life: towards a world free of tuberculosis. Geneva: World Health Organization, 2006. (WHO/HTM/ STB/2006.35).

6. World Health Organization. Global project on antituberculosis drug resistance surveillance: anti-tuberculosis drug resistance in the world. Fourth global report. Annex 9. Geneva: World Health Organization, 2008. 142 p. (WHO/HTM/TB/2008.394).

7. Franzblau SG, Witzig RS, McLaughlin JC, Torres P, Madico G, Hernandez A et al. Rapid, low-technology MIC determination with clinical Mycobacterium tuberculosis by using the microplate Alamar Blue assay. J Clin Microbiol 1998;36:362-6.

8. Roberts GD, Goodman NL, Heifets L, Larsh HW, Lindner TH, McClatchy JK et al. Evaluation of BACTEC radiometric method for recovery of mycobacteria and drug susceptibility testing of Mycobacterium tuberculosis from acid-fast smear-positive specimens. J Clin Microbiol 1983;18:689-96.

9. Palomino JC, Traore H, Fissette K, Portaels F. Evaluation of mycobacteria growth indicator tube (MGIT) for drug susceptibility testing of Mycobacterium tuberculosis. Int J Tuber Lung Dis 1999;3:344-8.

10. Birinci A, Coban AY, Ekinci B, Durupinar B. Comparison of the proportion method with mycobacteria growth indicator tube and E-test for susceptibility testing of Mycobacterium tuberculosis. Mem Inst Oswaldo Cruz 2002;97:351-2.

11. Bwanga F, Hoffner S, Haile M, Joloba ML. Direct susceptibility testing for multi drug resistant tuberculosis: a meta-analysis. BMC Infect Dis 2009;9:67.

12. Rossau R, Traore H, De Beenhouwer H, Mijs W, Jannes G, De Rijk P et al. Evaluation of the INNO-LiPA Rif. TB assay, a reverse hybridization assay for the simultaneous detection of Mycobacterium tuberculosis complex and its resistance to rifampin. Antimicrob Agents Chemother 1997;41:2093-8.

13. Golyshevskaia VI, Korneev AA, Chernousova LN, Selina LG, Kazarova TA, Grishina TD et al. [New 
microbiological techniques in diagnosis of tuberculosis]. Probl Tuberk 1996;6:22-5 [Russian].

14. Angeby KA, Klintz L, Hoffner SE. Rapid and inexpensive drug susceptibility testing of Mycobacterium tuberculosis with a nitrate reductase assay. J Clin Microbiol 2002;40:553-5.

15. Montoro E, Lemus D, Echemendia M, Martin A, Portaels F, Palomino JC. Comparative evaluation of the nitrate reduction assay, the MTT test, and the resazurin microtitre assay for drug susceptibility testing of clinical isolates of Mycobacterium tuberculosis. J Antimicrob Chemother 2005;55:500-5.

16. Sethi S, Sharma S, Sharma SK, Meharwal SK, Jindal SK, Sharma M. Drug susceptibility of Mycobacterium tuberculosis to primary antitubercular drugs by nitrate reductase assay. Ind J Med Res 2004;120:468-71.

17. Kumar M, Khan IA, Verma V, Kalyan N, Qazi GN. Rapid, inexpensive MIC determination of Mycobacterium tuberculosis isolates by using microplate nitrate reductase assay. Diagn Microbiol Infect Dis 2005; 53:121-4.

18. Kumar M, Khan IA, Verma V, Qazi GN. Microplate nitrate reductase assay versus Alamar Blue assay for MIC determination of Mycobacterium tuberculosis. Int J Tuberc Lung Dis 2005;9:939-41.

19. Poojary A, Nataraj G, Kanade S, Mehta P, Baveja S. Rapid antibiotic susceptibility testing of Mycobacterium tuberculosis: it's utility in resource poor settings. Ind J Med Microbiol 2006;24:268-72.

20. Mishra B, Muralidharan S, Srinivasa H. Direct drug susceptibility testing of Mycobacterium tuberculosis to primary anti-tubercular drugs by nitrate reductase assay. Ind J Pathol Microbiol 2009;52:343-4.

21. Visalakshi P, Meharwal SK, Myneedu VP, Behera D. Evaluation of direct method of drug susceptibility testing of Mycobacterium tuberculosis to rifampicin and isoniazid by nitrate reductase assay in a national reference laboratory. Diagn Microbiol Infect Dis 2010;66:148-52.

22. Centro Panamericano de Zoonosis. Tuberculosis bacteriology. Technical note 11. Buenos Aires: Centro Panamericano de Zoonosis, 1988 [Spanish].

23. Canetti G, Fox W, Khomenko A, Mahler HT, Menon NK, Mitchison DA et al. Advances in techniques of testing mycobacterial drug sensitivity, and the use of sensitivity tests in tuberculosis control programmes. Bull World Health Organ 1969;41:21-43.

24. Canetti G, Froman S, Grosset J, Hauduroy P, Langerova M, Mahler HT et al. Mycobacteria: laboratory methods for testing drug sensitivity and resistance. Bull World Health Organ 1963;29:565-78.

25. Altman DG. Practical statistics for medical research. In: Altman DG, editors. Inter-rater agreement. London: Chapman and Hall/CRC, 1999:403-9.

26. Martin A, Panaiotov S, Portaels F, Hoffner S, Palomino JC, Angeby K. The nitrate reductase assay for the rapid detection of isoniazid and rifampicin resistance in Mycobacterium tuberculosis: a systematic review and meta-analysis. J Antimicrob Chemother 2008;62:56-65.

27. Coban AY, Birinci A, Ekinci B, Durupinar B. Drug susceptibility testing of Mycobacterium tuberculosis with nitrate reductase assay. Int J Antimicrob Agents 2004;24:304-6.

28. Laszlo A, Rahman M, Espinal M, Raviglione M; WHO/IUATLD Network of Supranational Reference Laboratories. Quality assurance programme for drug susceptibility testing of Mycobacterium tuberculosis in the WHO/IUATLD Supranational Reference Laboratory Network: five rounds of proficiency testing, 1994-1998. Int J Tuberc Lun Dis 2002;6:748-56.

29. Martin A, Montoro E, Lemus D, Simboli N, Morcillo $\mathrm{N}$, Velasco M et al. Multicenter evaluation of the nitrate reductase assay for drug resistance detection of Mycobacterium tuberculosis. J Microbiol Methods 2005;63:145-50.

30. Martin A, Palomino JC, Portael F. Rapid detection of ofloxacin resistance in Mycobacterium tuberculosis by two low-cost colorimetric methods: resazurin and nitrate reductase assays. J Clin Microbiol 2005;43:16126.

31. Lemus D, Montoro E, Echemendía M, Martin A, Portaels F, Palomino JC. Nitrate reductase assay for detection of drug resistance in Mycobacterium tuberculosis: simple and inexpensive method for low-resource laboratories. J Med Microbiol 2006;55:861-3.

32. Khan A, Sarkar D. A simple whole cell based high throughput screening protocol using Mycobacterium bovis BCG for inhibitors against dormant and active tubercle bacilli. J Microbiol Methods 2008;73:62-8.

33. Musa RH, Ambroggi M, Souto A, Angeby KA. Drug susceptibility testing of Mycobacterium tuberculosis by a nitrate reductase assay applied directly on microscopy-positive sputum samples. J Clin Microbiol 2005;43:3159-61.

34. Shikama ML, Ferro e Silva R, Villela G, Sato DN, Martins MC, Giampaglia CM et al. Multicentre study of nitrate reductase assay for rapid detection of rifampicin-resistant M. tuberculosis. Int J Tuberc Lung Dis 2009;13:377-80.

35. Shikama Mde L, Silva RR, Martins MC, Giampaglia CM, Oliveira RS, Silva RF et al. Rapid detection of resistant tuberculosis by nitrate reductase assay performed in three settings in Brazil. J Antimicrob Chemother 2009;64:794-6.

36. Syre H, Phyu S, Sandvan P, Bjorvatn B, Grewal HM. Rapid colorimetric method for testing susceptibility of Mycobacterium tuberculosis to isoniazid and rifampin in liquid cultures. J Clin Microbiol 2003;41:5173-7.

37. Kent PT, Kubica GP. Public health mycobacteriology: a guide for the level III laboratory. Atlanta, GA: Centers for Disease Control, 1985:96-103. 